\title{
BLOCKCHAIN APPLICATION IN RFID DOMAIN
}

\section{Ivana Radović*, Nebojša Bačanin}

Singidunum University, Belgrade, Serbia

\begin{abstract}
:
Many people associate Blockchain technology exclusively with the payment system and earnings via the Internet, but its application is much greater. In this paper, after exploring chapters related to blockchain technology and microchips (RFID chips), we will be able to see how wide the application of blockchain technology is and the full extent of its capacity and possibilities for use in a variety of different areas of life. The paper aims to show the possibility of applying blockchain technology for object tracking. The idea of the application in a retail chain has been proposed.
\end{abstract}

Keywords:

Blockchain, RFID, chip, tag, reader.

\section{INTRODUCTION}

Mankind has always needed to have accurate information about the origin and history of any valuable object. Nowadays, however, as technology advances and the number of hackers increases, the number of counterfeit goods has grown and become easily available in the supply chain all over the world. Where the prevalence of the product is high, proof of the authenticity and the integrity of the goods is becoming increasingly difficult to prove. A possible solution to this problem is reflected in the application of blockchain technology to the source of and authenticity of many goods/products [1]

Combining blockchain technology with RFID chips gives us the ability to keep the data stored in the chip permanently, unchanged, and easy to read. RFID technology is characterized as reliable and can be easily adapted to the user's needs. 


\section{BLOCKCHAIN}

To better understand this new technology of marking and tracking products, we need to understand what a blockchain system is. This system can be described as one in which a record of transactions/information is maintained across several computers that are linked in a peer-to-peer network. A blockchain is a constantly growing ledger that keeps a permanent record of all the transactions that have taken place in a secure chronological and immutable way [1].

It is a database that is shared among network participants. It was created by connecting a P2P network (peer to peer) and a distributed server, which allows all transactions to be time-marked. This database is not stored in one central location (server), alternatively, it is distributed (decentralized) and represents a public book (Ledger) where all transactions that have ever occurred in respect of the product or goods are recorded and stored online. The database is public (rather than privately owned) and effectively is owned by all participants in the network [2].

The system of approval and acceptance of what is valid/accurate or not is based on group trust and consensus. Since the system is decentralized, all transactions and information contained in the ledger must be unchanged or agreed upon before any changes can occur. Data immutability is the essence of the system and the blockchain system itself differs from classic private databases, where changes and deletions of data are allowed [2].

As the information is stored in blocks (these blocks are put together to form a chain) and their distribution is done via the Internet where all nodes in the network have access to the blockchain itself. Nodes effectively form the infrastructure of a blockchain. All nodes on a blockchain are connected and they constantly exchange the latest blockchain data with each other so that all the nodes stay up to date with the same correct information and data. They store, spread and preserve the blockchain data, so theoretically a blockchain exists on nodes [2].

By allowing these nodes to access the blocks, all parties can get validation at any time of the information contained within that particular block. The first block in any blockchain is called the genesis block and each block in the chain contains the hash value of that data as well as the hash value of the previous transaction [2].

The securitisation and protection of the blockchain system itself is done by applying cryptographical methods and algorithms. For the blockchain system to function, cryptographical mechanisms are used:
- Hash functions;

- Public and private keys;

- Digital signing.

The inability to change the data contained within the blockchain is provided by the hash function and the consensus of the nodes in the network is required for any validation of the block. The immutability of data leads to trust in the system because it is difficult for any single individual or group of people to influence the data in any way. Data changes can occur only under the condition that most nodes in the network are represented by a group that wants to forcibly change the data, which is difficult to achieve [2].

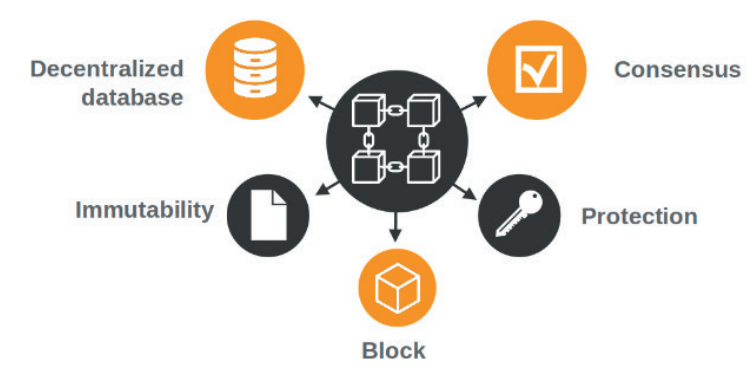

Figure 1 Blockchain technology architecture

\section{MICROCHIPS}

Microchips (chips) are widely used today, we can find them in computers, telephones, they have also been used for tracking pets. Scientists are still developing microchips, researching their applications, and today, microchips are produced that are barely noticeable to the human eye. The chip can receive millions of bits, which are usually grouped in groups of eight bytes [3].

Memory chips play a basic role in storing data that a microprocessor can access immediately. Since memory chips are mainly used in computers, we can divide them into two types [3]:

- RAM (Random Access Memory) - also known as the internal memory is where the data on a computer remains stored until the computer shuts down. There are two types of RAM as follows:

- SRAM (Static Random Access Memory);

- DRAM (Dynamic Random Access Memory); 
- ROM (Read Only Memory) - is data stored in memory that is not subject to change. Flash memory is a removable ROM memory, the data on it remains stored even after the computer is shut down. To remove data from ROM memory, developers must to reprogram parts of the chip.

\subsection{RFID CHIPS}

Radio Frequency Identification (RFID) is a technology that is highly developed today and is one of the most commonly used technologies. It allows the identification of objects, without the need for physical or visual contacts, using radio waves. The critical parameters of the identification process are the memory space and the time required for identification [4].

The RFID system can be represented by two components:

- Transceiver (transmitter / receiver) and antenna this combination is an RFID reader;

- Transponder (transmitter) and antenna represent RFID tag.

Communication between the tag and the reader is done using radio signals that transmit data. Data transmission can be one-way or two-way [5].

The data used in RFID technology is not strictly defined, which allows users to customize it to their own needs. These can be some measurements of the environment, products, timestamps, location of the facility... RFID technology can also be used in combination with cryptographical methods to increase data security [5].

\subsection{RFID TAGS AND READERS}

The reader has the role of delivering the downloaded information from the label to the computer in digital form. It consists of an antenna and a decoder. Readers can be portable (handheld terminals) or fixed [6].

An RFID tag can be represented as a small piece of material made up of an antenna and a microchip. Used to store data. Labels can be read-only or read-write, depending on the user's needs [5]. There are two types of labels [6]:

- Passive RFID tag does not have its own energy source, it relies on energy emitted by the reader.

- The active RFID tag has its own power source that they use to generate signals to respond to the reader. They are connected to the propulsion infrastructure or use the energy stored in the integrated battery. Active tags contain not only the battery but also some form of transmitter on the tag.

\subsection{RFID CHIPS}

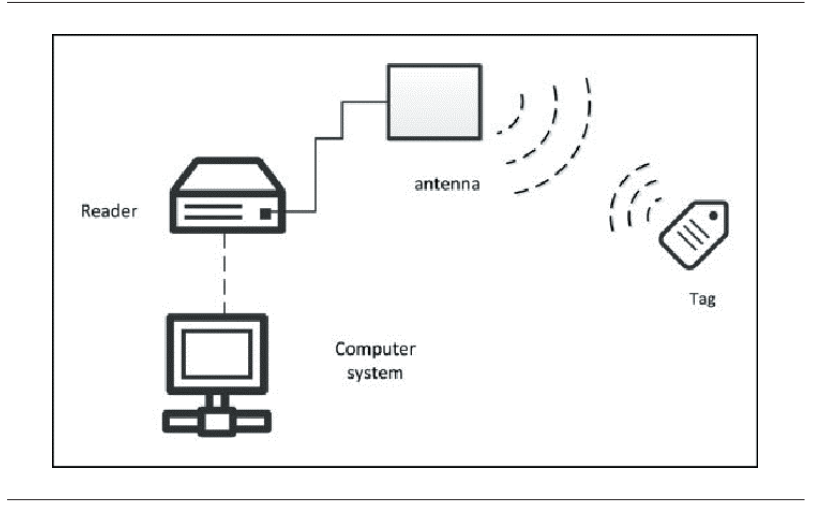

Figure 2 Working principle of RFID system [7]

The principle of operation of the RFID system is shown in Figure 2. When the tag reaches the reading zone, its data is transmitted to the reader via the antenna and the antenna transmits radio signals and thus activates the mark.

When contact is established between the antenna and the tag, data in the tag can be written or read.

When the RFID tag passes through the electromagnetic zone, a reader activation signal appears and it then decodes the data encoded in the integrated circuit tag (silicon chip) and transmits it to the computer. The data stored in the tag can identification data, location data, specific product data, et cetera.

RFID quickly attracted attention because it can be used to track moving objects [6].

\section{A COMBINATION OF RFID AND BLOCKCHAIN}

By combining blockchain technology with RFID technology, it is possible to achieve real-time monitoring of objects. The role of blockchain technology in this combination is reflected in the security and accuracy of data. The RFID system is used for the purpose of fast and accurate data transmission.

By combining these technologies, we can create supporting documentation that will be difficult to falsify. It is recommended to use multiple signatures, which prevent one party from independently creating records and changing the information entered in the documentation [5]. 
Smart contracts are programs that are stored on a chain of blocks and run when pre-set conditions are met and they are usually used to automate the execution of agreements so that all participants can be immediately sure of the outcome, without any involvement of intermediaries or waste of time. In fact, it is a collection of code and data located at a specific address on a chain of blocks [8]. Smart contracts in a combination of blockchain and RFID systems are also called triggers.

Automatic or semi-automatic data entry leads to time savings, increased accuracy, reduced labor needs, increased trust among users, and reduced false information. Time data and precise locations can be entered automatically [5].

An example of using a combination of these technologies can be found in a retail chain. The goal is to ensure trust between the buyer and the seller so that the buyer will have access to all relevant information about the desired product. Buyer can find out the place and time of production, delivery conditions, method of storage, information about the supplier, distributor,... [5] Through this example of application it is possible to avoid the purchase of counterfeit, unverified and unreliable products.

However, the actual application of RFID technology and blockchain is still limited due to technical and economic barriers [5].

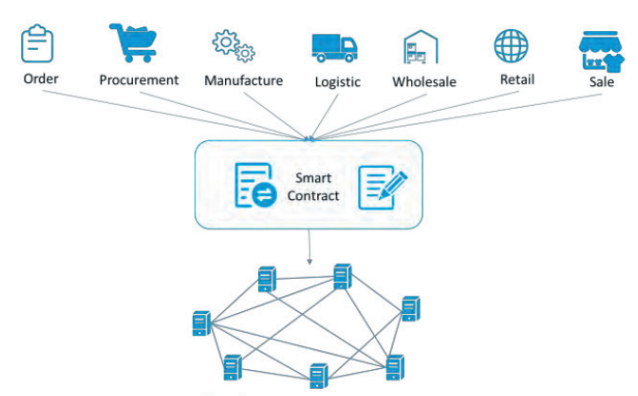

Fig. 3 Clothing Logistics Management

Figure 3 A retail chain that used RFID technology and blockchain [9]

A logistics management system based on the application of blockchain and RFID technology can be represented through a four-layer structure including an application layer, a blockchain data processing layer, and a data collection layer.

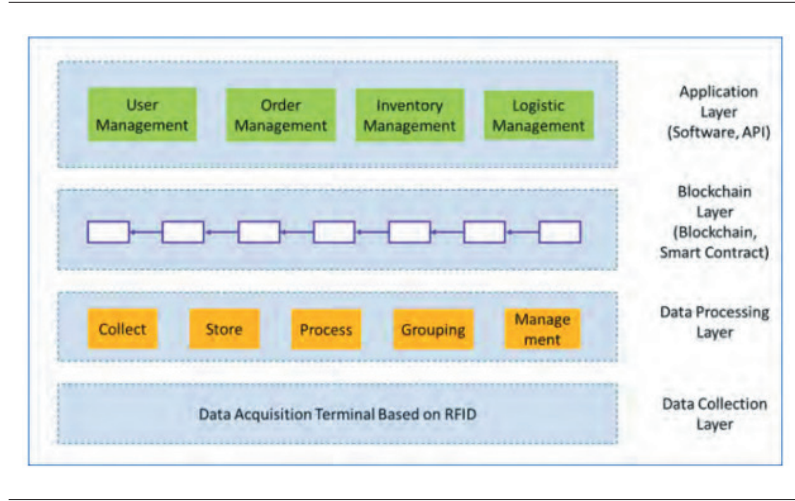

Figure 4 Logistics management system architecture [9]

The data collection layer is based on the application of RFID technology, which means that all data processed in the system are collected from objects that have an RFID tag and they are transmitted to a computer using a reader [9].

A data processing layer is also required. It is responsible for collecting, processing, transmitting, verifying the collected data, filtering information, removing redundant data (duplicates),... Data is first processed and then transferred to the blockchain layer [9].

The role of the blockchain system is reflected in the storage of data, but also the entire system. It enables the use of smart contracts that are built into the system and enables their execution regardless of external factors [9].

The application layer manages software functions, inventory, processes, and distributes orders. It is responsible for the user interface [9].

\section{CONCLUSION}

The first blockchain however was originally conceptualized by a person (or group of people) known as Satoshi Nakamoto who significantly improved the design by using a new methodology to time stamp blocks without requiring them to be signed by a trusted party and introducing a difficulty parameter to stabilize rate with which blocks are added to the chain. The design was implemented as a core component of the cryptocurrency bitcoin where it serves as the public ledger for all blockchain transactions on the network.

Its potential, however, has extended beyond financial currency usage and does not exclusively exist in that field. Using this system in combination with other technologies opens up new possibilities for its application. Specifically, this paper discussed the combination of blockchains with RFID chips for tracking an object or product, which provides security to system users. 
The convenience of using RFID technology is reflected in the high degree of reliability of reading data from the tag, the ability to read data if the reader and the tag do not have direct contact with each other Blockchain in this combination of technologies provides data security, ensures that all set conditions (if any) are met.

\section{REFERENCES}

[1] G. Levy, "Blockchain and Bitcoin Fundamentals".

[2] S. Adamović, "Bezbednosni mehanizmi i koncepti blockchain arhitekture,” Belgrade, 2020.

[3] J. Haack, E. Heller, B. Lee, E. Robinson, A. Wogsland, J. Yuma, "Electrifying Microchips".

[4] V. Tomašević, M. Tomašević, “Time-memory Trade-off in RFID Systems," in Sinteza, Belgrade, Serbia, 2016.

[5] L. KUBÁČ, "RFID TECHNOLOGY AND BLOCKCHAIN IN SUPPLY CHAIN,” Ostrava, 2018.

[6] M. S. N. M. a. P. S. Mandeep Kaur, "RFID Technology Principles, Adventages, Limitations \& Its Applications," International Journal of Computer and Electrical Engineering, 1 February 2010.

[7] "ReasearchGate," [Online]. Available: https:// www.researchgate.net/figure/Working-principleof-RFID-system_fig2_312442723?fbclid=IwAR3R DroO9CCsaSrrtz-5yTeRkQRSewx9dQgrlmtlBoQSm1Hrj_aNyru7wvA.

[8] “BINANCE Academy," 2018. [Online]. Available: https://academy.binance.com/en/articles/historyof-blockchain..

[9] R. Wang, W. Tsai, J. He, C. Liu, Q. Li and E. Deng, "Logistics Management System Based on Permissioned Blockchains and RFID Technology," in International Conference on Computer, Network, Communication and Information Systems, 2019. 